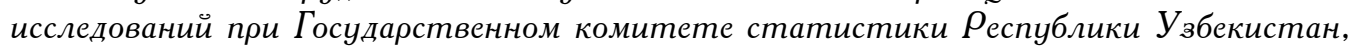
e-mail:tniyazmatov@mineconomy.uz

\title{
ВАИЯНИЕ ПИИ НА ЭКОНОМИЧЕСКИЙ РОСТ В РАЗВИВАЮЩИХСЯ СТРАНАХ И СТРАНАХ С ПЕРЕХОАНОЙ ЭКОНОМИКОЙ: ВАЖНОСТЬ ПОРОГОВОГО УРОВНЯ ПОГАОЩАЮЩЕЙ СПОСОБНОСТИ СТРАНЫ
}

\begin{abstract}
Аннотауия. Цель работы. Целью работы является определение, могут ли прямые иностранные инвестищии (ПИИ) сами по себе оказывать прямое (положительное) влияние на рост производства или эффект роста прямых иностранных инвестиций, что зависит от порогового уровня поглощающей способности принимающей экономики. Метод или методология проведения работы. Исследование основывается на построенной модели ПИИ, состоящей из различных переменных, которые могут оказывать положительное / отрииательное влияние на экономический рост, а затем на анализе результатов различных переменных. Результаты. Как показали эмпирические данные, влияние ПИИ на рост зависит от образованной рабочей силь, степени открытости торговли, развития инфраструктуры и уровня качества институтов. Политические стратегии, направленные на привлечение ПИИ, должны быть связаны с политикой, способствующей лучшей свободе экономической деятельности, а не предшествовать ей, поскольку большая свобода может принести большую выгоду. Область применения результатов.

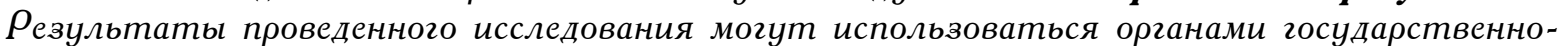
$2 о$ управления, министерствами и ведомствами различных стран. Выводы. В результате исследования были сделаны следующие выводы. Во-первых, как и в более ранних исследованиях, было установлено, ито сами ПИИ не оказывают прямого влияния на рост производства. Во-вторых, внутренние инвестищии, государственные расходы, человеческий капитал, открытость экономики принимающей страны (торговля), развитие инфраструктуры и институтов были признаны важными факторами долгосрочного роста. Ключевые слова: теория нового роста, накопление капитала, трансфер технологий, уровень открытости, поглощающиая способность, развитие человеческого капитала.
\end{abstract}

MAMATKULOV ILKHOM ABDURASHIDOVICH

research fellow of the Institute for advanced training and statistics research at the State statistics Committee of the Republic of Uzbekistan, e-mail:tniyazmatov@mineconomy.uz

\section{IMPACT OF FDI ON ECONOMIC GROWTH IN DEVELOPING COUNTRIES COUNTRIES AND COUNTRIES WITH ECONOMIES IN TRANSITION: THE IMPORTANCE OF THRESHOLD LEVEL OF THE COUNTRY'S ABSORPTION CAPACITY}

\begin{abstract}
Purpose of work. The purpose of the work is to determine whether foreign direct investment (FDI) itself can have a direct (positive) impact on the growth of production or the effect of the growth of foreign direct investment, which depends on the threshold level of absorption capacity of the host economy. Method or methodology of the work. The study is based on a constructed model of FDI consisting of various variables that can have a positive / negative impact on economic growth, and then on the analysis of the results of various variables. Results. Empirical evidence has shown that the impact of FDI on growth depends on the educated workforce, the degree of openness to trade, infrastructure development, and the quality of institutions.

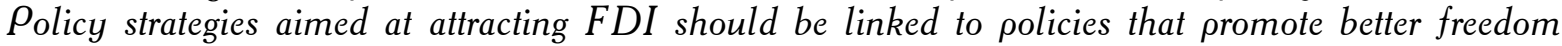
of economic activity, rather than preceding it, since greater freedom can bring greater benefits. The scope of the results. The results of the study can be used by public authorities, ministries and departments of different countries. Summary. As a result of the study, the following conclusions
\end{abstract}




\section{МАМАТКУЛОВ И.А.}

ВЛИЯНИЕ ПРЯМЫХ ИНОСТРАННЫХ ИНВЕСТИЦИЙ НА ЭКОНОМИЧЕСКИЙ РОСТ В РАЗВИВАЮЩИХСЯ СТРАНАХ И СТРАНАХ С ПЕРЕХОДНОЙ ЭКОНОМИКОЙ: ВАЖНОСТЬ ПОРОГОВОГО УРОВНЯ ПОГЛОЩАЮЩЕЙ СПОСОБНОСТИ СТРАНЫ were made. First, as in earlier studies, it was found that FDI itself did not have a direct impact on output growth. Second, domestic investment, public spending, human capital, the openness of the host country's economy (trade), and the development of infrastructure and institutions were recognized as important factors for long-term growth.

Keywords: theory of new growth, capital accumulation, technology transfer, level of openness, absorbing capacity, human capital development.

В данной статье приводятся доказательства, что некоторые из принципов новой теории роста (Pack, 1994) [14] обеспечивают соответствующую основу для определения наблюдаемой эффективности прямых иностранных инвестиций в стимулировании роста в странах, придерживающихся либеральных режимов внешней торговли и инвестиций, достаточного уровня человеческого капитала и заслуживающего доверия институтов, обеспечивающих понимание характеристик среды, благоприятствующей процветанию. В этом контексте будет определен ряд проверяемых гипотез.

Суть новой теории роста заключается в том, что вопреки неоклассической мудрости рост может быть эндогенным. В неоклассических моделях, таких как Солоу (1956) [17], воплощающих постоянную отдачу от масштабных функций и стандартное поведение максимизации полезности, увеличение накопления капитала приведет к уменьшению отдачи от капитала. Это, конечно, препятствует как сбережениям, так и инвестициям. Учитывая такие убывающие доходы, рост может происходить только при наличии рабочей силы или технических изменений. Оба эти источника являются экзогенными: первый изменяет наклон производственной функции, а второй смещает его наружу. Кроме того, модели экстентов не дают объяснения ни для технических изменений, ни для роста, связанного с термином А в знакомой производственной функции типа Кобба-Дугласа.

Эндогенный рост является результатом механизмов, которые предотвращают неограниченное снижение предельного физического продукта капитала [3]. Это может произойти из-за растущего возврата к масштабу, несовершенной конкуренции и накоплению человеческого капитала или побочных эффектов. Несмотря на то, что существует множество моделей, большинство из них подчеркивают эффекты распространения человеческого капитала и знаний или внешние эффекты, присущие исследованиям и разработкам (НИОКР) [10]. Одна идея заключается в том, что, хотя частные нормы прибыли на инвестиции в НИОКР могут со временем снижаться, социальная норма прибыли может быть высокой [20]. Расхождение между социальными и частными показателями доходности инвестиций является общей чертой этих моделей. Именно высокая социальная норма прибыли за счет внешних факторов дает основание для снижения предельного продукта капитала в совокупности. Другая идея заключается в том, что отдача от накопления человеческого капитала не соответствует убывающей отдаче так же, как отдача от накопления физического капитала. Такие инвестиции увеличивают не только частную, но и социальную прибыль [18].

Новая теория роста четко выделяет те факторы, которые должны характеризовать ПИИ. В той степени, в которой считается, что прямые иностранные инвестиции передают технологии, способствуют обучению на практике, обучают труду и, как правило, приводят к распространению человеческих навыков и технологий, они должны способствовать росту [4]. Однако для того, чтобы все это произошло, должно быть выполнено несколько условий, и поэтому в статье предлагается следующая основная гипотеза.

ГИПОТЕЗА: ПИИ сами по себе могут не оказывать прямого (положительного) влияния на рост производства. Вместо этого эффект роста ПИИ зависит от порогового уровня поглощающей способности принимающей развивающейся экономики [19].

Приведенная выше гипотеза обеспечивает основу для объяснения эффектов ПИИ, способствующих росту в целом и в частности для наблюдаемой эффективности ПИИ в стимулировании роста в странах, действующих в контексте наличия определенного уровня поглощающей способности, например, квалифицированной рабочей силы, развития инфраструктуры, уровня открытости торгового режима, институционального качества и развития финансовой системы.

Для эмпирической оценки влияния ПИИ на экономический рост можно использовать сле- 
дующую формулировку:

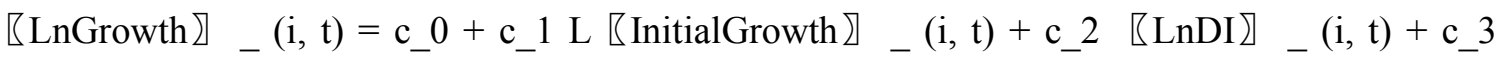

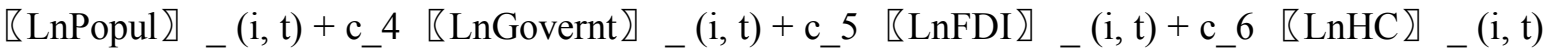
$+c_{-} 7$ 『LnInst _ $(\mathrm{i}, \mathrm{t})+\mathrm{c} \_8$ 『LnTrade _ $(\mathrm{i}, \mathrm{t})+\mathrm{c} \_9$ 『LnInfras 】 $(\mathrm{i}, \mathrm{t})+\mathrm{c} \_10$ 『LnFinance 】_ $(\mathrm{i}, \mathrm{t})+\mathrm{c} \_11(\operatorname{LnFDI} \times \operatorname{LnABS})_{-}(\mathrm{i}, \mathrm{t})+\mathrm{n}_{-} \mathrm{i}+\varepsilon_{-}(\mathrm{i}, \mathrm{t})(1)$

Эта модель использовалась для проверки изложенных выше гипотез традиционным образом из производственной функции, в которой ПИИ вводятся в качестве входных данных в дополнение к труду и внутреннему капиталу. ПИИ можно рассматривать в качестве основного источника человеческого капитала и новых технологий для развивающихся стран, и эта переменная включена в производственную функцию для определения внешних факторов, обучения посредством наблюдения и побочных эффектов, связанных с ПИИ [6]. Более того, рост численности населения и государственные расходы были введены в качестве дополнительного фактора, влияющего на производственную функцию, следуя теории и большому количеству эмпирических исследований (например, Alfaroetal, 2004; [1] Durham, 2004; [7] Azmanetal, 2010) [2]. После проверки линейности функции, взятия логов и дифференцирования было получено приведенное выше выражение, описывающее детерминанты темпов роста ВВП. Ввиду хорошо известных и серьезных проблем, связанных с попытками измерить запас капитала, особенно в контексте развивающихся стран, он последовал прецеденту, установленному во многих предыдущих исследованиях, путем аппроксимации темпов роста капитала за счет доли инвестиций в ВВП.

Таблий 1

Абсорбционные возможности и влияние ПИИ на экономический рост; 1997-2011 (двухступенчатая системная модель GMM, зависимая переменная: темпы роста реального ВВП)

\begin{tabular}{|c|c|c|c|c|c|c|c|}
\hline & 1 & 2 & 3 & 4 & 5 & 6 & 7 \\
\hline Initial LGROWTH & $\begin{array}{c}-0,178^{* * * *} \\
0,098\end{array}$ & $\begin{array}{c}-0,277^{* *} \\
0,088\end{array}$ & $\begin{array}{c}-0,159^{* * * *} \\
0,063\end{array}$ & $\begin{array}{c}-0,262^{* * * *} \\
0,051\end{array}$ & $\begin{array}{c}-0,173^{* * *} \\
0,088\end{array}$ & $\begin{array}{c}-0,291 * * * \\
0,086\end{array}$ & $\begin{array}{c}-0,268 * * * \\
0,061\end{array}$ \\
\hline LDI & $\begin{array}{c}0,361^{* * *} \\
0,074 \\
\end{array}$ & $\begin{array}{c}0,370^{* * *} \\
0,065\end{array}$ & $\begin{array}{c}0,396 * * * \\
0,063 \\
\end{array}$ & $\begin{array}{c}0,503 * * * \\
0,061 \\
\end{array}$ & $\begin{array}{c}0,438 * * * \\
0,058\end{array}$ & $\begin{array}{c}0,367 * * * \\
0,062 \\
\end{array}$ & $\begin{array}{c}0,451^{* * *} \\
0,142\end{array}$ \\
\hline LFDI & $\begin{array}{c}-0,113 * * * \\
0,041\end{array}$ & $\begin{array}{c}-0,595 * * * \\
0,233\end{array}$ & $\begin{array}{c}0,397 * * * \\
0,14\end{array}$ & $\begin{array}{c}-0,476^{* *} \\
0,301\end{array}$ & $\begin{array}{c}-0,059 * * \\
0,028\end{array}$ & $\begin{array}{c}-0,091 * * \\
0,095\end{array}$ & $\begin{array}{c}-1,13 * * \\
1,037\end{array}$ \\
\hline LPOPUL & $\begin{array}{l}0,064 \\
0,098\end{array}$ & $\begin{array}{l}0,074 \\
0,099\end{array}$ & $\begin{array}{l}0,041 \\
0,066\end{array}$ & $\begin{array}{c}-0,045 \\
0,081\end{array}$ & $\begin{array}{c}-0,119 * * \\
0,058\end{array}$ & $\begin{array}{c}-0,100^{*} \\
0,053\end{array}$ & $\begin{array}{c}-0,04 \\
0,08\end{array}$ \\
\hline LGOVERNT & $\begin{array}{c}-0,248^{*} \\
0,142\end{array}$ & $\begin{array}{c}-0,252^{*} \\
0,129\end{array}$ & $\begin{array}{c}-0,253^{*} \\
0,134\end{array}$ & $\begin{array}{c}-0,358^{* * *} \\
0,129\end{array}$ & $\begin{array}{c}-0,316^{* *} \\
0,141\end{array}$ & $\begin{array}{c}-0,207 * * \\
0,094 \\
\end{array}$ & $\begin{array}{c}-0,268^{*} \\
0,154\end{array}$ \\
\hline LHC & $\begin{array}{c}0,184 * * \\
0,071\end{array}$ & $\begin{array}{c}-0,215^{*} \\
0,123\end{array}$ & $\begin{array}{c}0,139 * * \\
0,062\end{array}$ & $\begin{array}{c}0,196 * * \\
0,095\end{array}$ & $\begin{array}{c}0,306 * * * \\
0,068\end{array}$ & $\begin{array}{c}0,250 * * * \\
0,061\end{array}$ & $\begin{array}{c}-1,371^{*} \\
0,066\end{array}$ \\
\hline LFDI*LHC & & $\begin{array}{c}0,139 * * \\
0,052\end{array}$ & & & & & $\begin{array}{c}0,506^{* *} \\
0,025\end{array}$ \\
\hline LINST & & & $\begin{array}{c}0,482 * * * \\
0,027\end{array}$ & & & & $\begin{array}{c}0,746^{* *} \\
0,032\end{array}$ \\
\hline LFDI*LINST & & & $\begin{array}{c}-0,122 * * * \\
0,038\end{array}$ & & & & $\begin{array}{c}-0,212 \\
0,112\end{array}$ \\
\hline LTRADE & & & & $\begin{array}{l}-0,11 \\
0,181\end{array}$ & & & $\begin{array}{c}-0,184 \\
0,269\end{array}$ \\
\hline LFDI*LTRADE & & & & $\begin{array}{c}0,125^{*} \\
0,069\end{array}$ & & & $\begin{array}{c}0,007 * * \\
0,034\end{array}$ \\
\hline LINFRAS & & & & & $\begin{array}{c}-0,045^{* *} \\
0,019\end{array}$ & & $\begin{array}{c}-0,044 \\
0,059\end{array}$ \\
\hline LFDI*LINFRAS & & & & & $\begin{array}{l}0,005 \\
0,006\end{array}$ & & $\begin{array}{c}0,021 * * \\
0,018\end{array}$ \\
\hline LFINANCE & & & & & & $\begin{array}{c}-0,186^{* *} \\
0,078\end{array}$ & $\begin{array}{l}0,096 \\
0,282\end{array}$ \\
\hline
\end{tabular}


МАМАТКУЛОВ И.А.

ВЛИЯНИЕ ПРЯМЫХ ИНОСТРАННЫХ ИНВЕСТИЦИЙ НА ЭКОНОМИЧЕСКИЙ РОСТ В РАЗВИВАЮЦИХСЯ СТРАНАХ И СТРАНАХ С ПЕРЕХОДНОЙ ЭКОНОМИКОЙ: ВАЖНОСТЬ ПОРОГОВОГО УРОВНЯ ПОГЛОЩАЮЩЕЙ СПОСОБНОСТИ СТРАНЫ

Продолюение таблицы 1

\begin{tabular}{|l|c|c|c|c|c|c|c|}
\hline & 1 & 2 & 3 & 4 & 5 & 6 & 7 \\
\hline LFDI*LFINANCE & & & & & & $\begin{array}{c}0,022 \\
0,026\end{array}$ & $\begin{array}{c}0,079 \\
0,092\end{array}$ \\
\hline Constant & $\begin{array}{c}1,838^{* * *} \\
0,522\end{array}$ & $\begin{array}{c}3,688^{* * *} \\
0,469\end{array}$ & $\begin{array}{c}0,177^{* *} \\
0,391\end{array}$ & $\begin{array}{c}2,602 * * * \\
0,827\end{array}$ & $\begin{array}{c}1,494 * * * \\
0,502\end{array}$ & $\begin{array}{c}1,920^{* * *} \\
0,404\end{array}$ & $\begin{array}{c}3,338^{* *} \\
2,645\end{array}$ \\
\hline Threshold Value & - & 4,28 & 3,25 & 3,81 & - & - & - \\
\hline Observations & 686 & 686 & 686 & 686 & 686 & 686 & 686 \\
\hline Instrumental Variables & 37 & 41 & 45 & 46 & 45 & 45 & 73 \\
\hline $\begin{array}{l}\text { P-Arellano-Bond test for AR } \\
\text { (2) in first diff. }\end{array}$ & 0,144 & 0,143 & 0,149 & 0,187 & 0,172 & 0,152 & 0,168 \\
\hline $\begin{array}{l}\text { P-Hansen test for over id. } \\
\text { Restrictions }\end{array}$ & 0,27 & 0,234 & 0,5 & 0,103 & 0,518 & 0,306 & 0,988 \\
\hline $\begin{array}{l}\text { Diff. in Hansen test of exoge- } \\
\text { neity of instr. subsets }\end{array}$ & 0,381 & 0,372 & 0,577 & 0,26 & 0,574 & 0,28 & 921 \\
\hline
\end{tabular}

Standard errors reported in parentheses. The system includes a time dummy variable to account for period-specific effects. $* * *, * *$ denote significance at $10 \%, 5 \%$, and $1 \%$, respectively. Region and time dummies are applied in the analysis. As HC, Trade, Inst, Infras and Finance used as proxies for absorptive capacity of the host countries, to avoid the possibility of there being correlated, the variable and their interaction effects enter the regression equation one-by-one.

Колонка 3 также подтверждает гипотезу о том, повышает ли качество институтов потенциальную выгоду ПИИ для экономического роста. В соответствии со значительным, но с неожиданным признаком срока взаимодействия индекса экономической свободы с ПИИ, можно упомянуть, что прямые иностранные инвестиции оказывают положительное влияние на рост, и этот эффект уменьшается с улучшением поглощающей способности принимающей страны. Расчетный порог для индекса экономической свободы составляет 3,25 , поэтому практически положительное влияние ПИИ на экономический рост будет сохраняться в принимающих странах до этого минимального порога. Важно отметить, что подобные результаты были получены Даремом (2004). Дарем утверждал, что, несмотря на отрицательный коэффициент взаимодействия, учитывая положительную и статистически значимую стоимость ПИИ, страны с более благоприятными правами собственности и рейтингом коррупции в среднем имеют более высокие темпы роста.

Гипотеза о том, что связь между внутренними запасами ПИИ и экономическим ростом зависит от торговли, также была подтверждена, как показано в столбце 4. Кроме того, это показывает, что открытость торговли существенно и положительно связана с экономическим ростом, подтверждая эмпирические исследования, анализирующие влияние открытости торговли для экономического роста, такие как Balasubramanyametal (1996), Яниккая (2003) и Макки и Сомвару (2004). Степень открытости является индикатором, который отражает уровень открытия местного рынка, так что более высокая степень открытости часто связана с большей рыночной дисциплиной и дополнительными точками продаж товаров и услуг, производимых отечественными фирмами [15]. Столбец показывает, что порог степени открытости равен 3,81 .

Столбцы 5 и 6 показывают положительное влияние ПИИ на экономический рост, основанное на их взаимодействии с инфраструктурой и переменными развития финансового рынка. В противоположность выводам KinishitaandLu (2006) [11] и Lumbilla (2005) [12] в этом документе не было обнаружено значения прямых иностранных инвестиций для экономического роста развивающихся стран благодаря этим двум способностям освоения. Однако, основываясь на положительном знаке условий взаимодействия, столбцы подтверждают гипотезу о том, что связь между ПИИ и ростом зависит от уровня инфраструктуры и развития финансового рынка, что предполагает, что принимающие страны должны достичь определенного уровня этих способностей освоения средств для извлечения выгоды из прямых иностранных инвестиций. Эти переменные были включены в анализ данной статьи после других эмпирических исследований, чтобы не столкнуться с пропущенным переменным смещением в оценке.

В столбце 7 представлено влияние всех наборов объясняющих переменных с их терминами взаимодействия на экономический рост. Как видно из последнего столбца таблицы, в исследо- 
вании достигнуты существенные эффекты практически всех независимых переменных с их ожидаемой корреляцией с зависимой переменной. Таким образом, полученные результаты этого исследования еще раз подтвердили результаты вышеупомянутых эмпирических исследований, указывающих на важную роль поглощающего потенциала в полном использовании преимуществ ПИИ для экономического роста развивающихся стран. Другими словами, эти результаты можно интерпретировать как свидетельство того, что воздействие ПИИ на экономический рост в значительной степени зависит от изменений в применяемых методах. Результаты также могут быть истолкованы как предложение исходной поддержки гипотезы о том, что внутренние запасы ПИИ могут оказать негативное влияние на экономический рост, и страны, которые предлагают всепоглощающий потенциал, получают наибольшую выгоду от внешних эффектов ПИИ [13]. В дополнение к результатам в предыдущих столбцах последний столбец подтвердил еще одну гипотезу о том, что вклад ПИИ в экономический рост зависит от уровня развития инфраструктуры, подтверждая предыдущие выводы эмпирических исследований, таких как Люмбила (2005), Киношита и Лу (2006).

Используя групповые данные по 49 развивающимся странам за период 1997-2011 годов, из эмпирического анализа был сделан ряд важных выводов. На основании результатов последнего столбца таблицы 6 можно сделать следующие выводы. Во-первых, как и в более ранних исследованиях, было установлено, что сами ПИИ не оказывают прямого влияния на рост производства [8]. Во-вторых, внутренние инвестиции, государственные расходы, человеческий капитал, открытость экономики принимающей страны (торговля), развитие инфраструктуры и институтов были признаны важными факторами долгосрочного роста [9].

Напротив, начальные темпы роста ВВП, рост населения, государственные расходы отрицательно связаны с экономическим ростом, что подтверждается исследованиями Каркович и Левина (2002) [5]. Наконец, как показали эмпирические данные, влияние ПИИ на рост зависит от образованной рабочей силы, степени открытости торговли, развития инфраструктуры и уровня качества институтов. Страны, в которых проводится обзор, могут значительно выиграть от присутствия ТНК, продвигая свободу экономической деятельности и улучшая качество человеческих ресурсов. В этих странах МНП могут легче освоить и принять новые технологии и другие преимущества, связанные с притоком ПИИ.

В этом отношении политики должны взвесить стоимость политики, направленной на привлечение ПИИ, по сравнению с теми, которые стремятся повысить уровень человеческого капитала, международные торговые отношения, развитие инфраструктуры и институтов [16]. Политические стратегии, направленные на привлечение ПИИ, должны быть связаны с политикой, способствующей лучшей свободе экономической деятельности, а не предшествовать ей, поскольку большая свобода может принести большую выгоду. Политики должны также создать политику, достаточно прозрачную для потенциальных инвесторов, прежде чем использовать другие меры для привлечения более высоких уровней ПИИ. Тем не менее реформы могут быть трудным процессом и требуют долгосрочных обязательств. В некоторых странах они могут быть политически трудными в краткосрочной перспективе, но долгосрочные экономические выгоды могут быть огромными.

\section{References:}

1. Alfaro L., Chanda A., Kalemli-Ozcan S. \& Sayek S. 2004. FDI and economic growth: the role of local financial markets // Journal of International Economics. Vol. 64. No. 1. P. 89-112.

2. Azman-Saini W., Baharumshah A. Z. \& Law S.H. 2010. Foreign direct investment, economic freedom and economic growth: International evidence // Economic Modelling. Vol. 27. No. 5. P. 1079-1089.

3. Barro R. J., Mankiw N. G. \& Sala-i-Martin X. 1992. Capital mobility in neoclassical models of growth. P. 128.

4. Calvet A. L. 1981. A synthesis of foreign direct investment theories and theories of the multinational firm // Journal of International Business Studies, P. 43-59.

5. Carkovic M. V.\& Levine R. 2002. Does foreign direct investment accelerate economic growth? // U of Minnesota Department of Finance Working Paper.

6. Carlsson F. \& Lundström S. 2002. Economic freedom and growth: Decomposing the effects, Public Choice. Vol. 112. No. 3-4. P. 335-344.

7. Durham J. B. 2004. Absorptive capacity and the effects of foreign direct investment and equity foreign portfolio investment on economic growth // European Economic Review. Vol. 48. No. 2. P. 285-306.

8. Fosfuri A., Motta M. \& Rønde T. 2001. Foreign direct investment and spillovers through workers' mobility /I Journal of International Economics. Vol. 53. No. 1. P. 205-222. 
МАМАТКУЛОВ И.А.

ВЛИЯНИЕ ПРЯМЫХ ИНОСТРАННЫХ ИНВЕСТИЦИЙ НА ЭКОНОМИЧСКИЙ РОСТ В РАЗВИВАЮЦИХСЯ СТРАНАХ И СТРАНАХ С ПЕРЕХОДНОЙ ЭКОНОМИКОЙ: ВАЖНОСТЬ ПОРОГОВОГО УРОВНЯ ПОГЛОЩАЮЩЕЙ СПОСОБНОСТИ СТРАНЫ

9. Grossman G. M. \& Helpman E. 1990. Trade, innovation, and growth // The American Economic Review. P. 86-91.

10. Herzer D. \& Klasen S. 2008. In search of FDI-led growth in developing countries: The way forward // Economic Modelling. Vol. 25. No. 5. P. 793-810.

11. Kinoshita Y. \& Lu C. 2006. On the role of absorptive capacity: FDI matters to growth.

12. Lumbila K. N. 2005. Risk, FDI and Economic Growth: A Dynamic Panel Data Analysis of the Determinants of FDI and Its Growth Impact in Africa.

13. Makki S. S. \& Somwar A. 2004. Impact of foreign direct investment and trade on economic growth: Evidence from developing countries // American Journal of Agricultural Economics. Vol. 86. No. 3. P. 795-801.

14. Pack H. 1994. Endogenous growth theory: intellectual appeal and empirical shortcomings // The Journal of Economic Perspectives. P. 55-72.

15. Schultz TW. 1961. Investment in human capital // The American Economic Review. P. 1-17.

16. Shin S. 1998. A Critical Review of the FDI Theories. Vol. 5. P. 179-211.

17. Solow R. M. 1956. A contribution to the theory of economic growth // The quarterly journal of economics. P. 65-94.

18. Spiezia V. 2004. Trade, foreign direct investment and employment: Some empirical evidence, Understanding Globalization, Employment and Poverty Reduction, ILO International Policy Group. London: Palgrave Macmillan.

19. Subasat T., Bellos S., (2002). Corruption and Foreign Direct Investment: A Panel Gravity Model Approach, Bulletin of Economic Research.

20. Vernon, Raymond (1966). International Investment and International Trade in the Product Cycle // The Quarterly Journal of Economicsю Vol. 80. P. 190-207. 